graphs are alternately taken by a $76 \mathrm{~mm}$ lens and a $25 \mathrm{~mm}$ lens. The vidicon is a specially designed rugged tube made by Radio Corporation of America.

The ground complex is the Deep Space Network and it includes $(a)$ three tracking stations located in California, South Africa, and Australia; $(b)$ the Space Flight Operations Facility located at the Jet Propulsion Laboratory in California; and (3) world-wide voice and teletype communications network. TV signals from the spacecraft are separated and recorded by the ground system on devices similar to commercial kinescopes used for filming TV programs. The signals are also recorded on magnetic tape.

Three highly-trained and specialized groups necessary to a space mission are (a) Spacecraft Data Analysis Team; (b) Flight Path Analysis and Command Group; and (3) Space Science Analysis Group.

All flight events occurred in normal sequence and at the proper time. A midcourse maneuver was performed which placed the impact only 6 miles from the target of $I^{\circ}$ south and $2 I^{\circ}$ west lunar selenocentric coordinates. No terminal orientation maneuver was required. The camera system returned over 4300 excellent photographs of the Moon during its $I_{7}$ minutes of operation prior to impact. The last photograph was taken at an altitude of approximately 500 meters. A surface resolution of approximately 0.5 meters was achieved. Cratering phenomena can be observed down to the limit of detectability in the photograph.

The tracking accuracy for Ranger VI and VII permitted refinement of the determination of the mass of both the Earth and the Moon.

Future Ranger missions will sample other mare areas to provide more information for the unmanned and manned lunar landing missions and increase our scientific knowledge of the Moon.

This paper presents the results of research carried out under contract NAS 7-Ioo sponsored by NASA.

\title{
3. RANGER VII CAMERA CALIBRATION AND PERFORMANCE
}

\author{
G. M. Smith, D. E. Willingham, W. H. Kirhofer \\ (Jet Propulsion Laboratory, California Institute of Technology, Pasadena, \\ California, U.S.A.)
}

This paper discusses factors which increase usefulness of the existing Ranger VII photographic system design.

A mathematical model utilizing a camera system description; a generalized lunar photometric function; a human observer model; and a trajectory/spacecraft description was developed and programed into a digital computer for the purpose of selecting optimum lunar aiming areas. The output of the program was considered a performance indicator (an estimate of the smallest surface feature observable on $35 \mathrm{~mm}$ film positive from the camera system). The specific subjects considered in the computer program analysis are discussed in the paper in detail.

Several problems related to the photographic system itself, including light transfer characteristic, modulation transfer function, and system noise, are described and solutions given.

A comprehensive description of the complete Ranger VII mission is presented with emphasis on the measures taken to ensure a photographic success. Specific figures are given for all significant data connected with each phase of the mission from launch to impact.

This paper presents the results of research carried out under contract NAS 7-IOo sponsored by NASA. 